\title{
Evolución legislativa de la educación vial en España: nuevos retos para educadores e instituciones
}

Ma Paz Trillo Miravalles*

\section{Resumen}

La educación vial ha sufrido variaciones a lo largo de los años. De una concepción restringida a aspectos vinculados a la circulación y las normativas, se ha derivado en una concienciación ciudadana que engloba aspectos legales, medioambientales, médicos, económicos, personales y convivenciales, entre otros. Desde el Día Mundial de la Salud celebrado en 2004 y los debates subsiguientes en la Asamblea General de las Naciones Unidas, los gobiernos se interesan cada vez más en la Seguridad Vial, incidiendo en la necesidad de la formación vial, como medio para reducir los accidentes de tráfico. La presente colaboración analiza la regulación vial en España, deteniéndose en la incorporación de la educación vial en el currículo y su evolución desde el Código de Circulación de 1934 hasta la Ley Orgánica de Educación, y ofrece nuevos desafíos y obligaciones a los que se enfrentan gobiernos, educadores e instituciones para tratar de frenar los elevados índices de siniestralidad en la carretera. Palabras clave: Educación. Vial. Legislación. Ciudadanía.

\section{Introducción}

Alrededor de 1,2 millones de personas mueren cada año en el mundo a causa de colisiones de tránsito y otras muchas, que apenas salen a la luz, sufren traumatismos. Son la segunda causa principal de muerte de las personas de 5 a 25 años, y tienen un impacto devastador en las familias y las comunidades.

Las legislaciones han transcurrido desde las limitadas al aprendizaje de las normas de circulación y las recomendaciones en relación a la seguridad vial, hasta las centradas en aspectos propios de una educación en valores, necesaria para crear actitudes positivas y tratar de erradicar la epidemia social generada por los accidentes de tráfico.

* Doctora por la Universidad Nacional de Educación a Distancia (UNED, España). Profesora en el Departamento de Métodos de Investigación y Diagnóstico en Educación I (UNED, España). E-mail: mptrillo@edu.uned.es 
Este estudio muestra la evolución legislativa sobre educación vial en España permitiendo conjugar ideas para elaborar normativas viales más enriquecedoras que puedan aplicarse de forma real y se sustenten en la educación. Por otra parte, ofrece claves a través de esas normativas, para ejercer una práctica profesional en educación vial más civilizada, crítica y de calidad tanto en España como en el resto de países.

La sociedad se ha visto obligada a regular el comportamiento de las personas mediante leyes y códigos, cuyo conocimiento es necesario para garantizar la convivencia. La incorporación de la educación vial en la legislación, viene siendo demandada por la sociedad, como elemento básico para abordar la problemática de los accidentes de tráfico y sus secuelas. El objetivo principal sería ofrecer una política duradera en la educación (WERLE; SOARES, 2008).

A través de la educación se asimilan, aprenden y generan conocimientos, normas de conducta, modos de ser y formas de ver el mundo. El educando se hace capaz de adquirir un pensamiento crítico. Los resultados de este proceso son una serie de habilidades, conocimientos, actitudes y valores adquiridos, desarrollando facultades intelectuales, emocionales, culturales, físicas y morales que producen cambios. Ello da origen a un pensamiento propio que facilita el razonamiento con una serie de ideas que crean la personalidad característica de cada sujeto, expresada en su comportamiento.

"Cada persona, al tiempo que va construyendo su personalidad moral, coparticipa de la construcción moral del entorno y, por ello, la integración de la dimensión individual y social de la educación debe encuadrarse en el marco de un modelo ético que contribuya a preservar al individuo, a la humanidad y a los demás seres vivos. Si hay algún tema que deba abordarse temprana y sistemáticamente desde la construcción de la personalidad moral y desde el objetivo de una seguridad vial sostenible, es el de la educación vial”. (JIMÉNEZ, 2010b, p.16).

Las normativas imponen una responsabilidad compartida entre las instituciones y los agentes educadores, junto a todos y cada uno de los usuarios de las vías, que reclaman diseños, actuaciones y evaluaciones compartidas y solidarias en favor de una movilidad más fluida y más segura (MANSO, 2010).

El análisis de los reglamentos establecidos en materia de educación vial genera un interés aún mayor por este artículo, debido a la escasez de publicaciones en la temática. 


\section{Desarrollo}

La evolución del término de educación vial se corresponde con los diversos enfoques y aproximaciones que desde la sociedad se han venido dando. En 1963 y 1971, en las conferencias celebradas en París y Viena por Instituciones internacionales como el "Consejo de Europa" y la "Conferencia Europea de Ministros de Transportes", se muestra su preocupación por la problemática del aumento de los accidentes de tráfico, fijando programas que contemplen la educación vial bajo el siguiente planteamiento:

- Velar por la propia seguridad y comportamiento de manera responsable, teniendo en consideración a los demás usuarios.

- Preparar a los futuros conductores de bicicletas, ciclomotores y automóviles.

- Informarse y utilizar los medios para mejorar la seguridad del sistema actual.

- Respetar todas las normas de circulación, incluso las que no son reglas de seguridad en sentido estricto.

El CIDE (Centro de Investigación y Documentación Educativa) dependiente del Ministerio de Educación español incide en el aspecto socializador de la educación vial.

"La educación vial es imprescindible para conseguir una educación ciudadana integradora de todos los principios que fomenten la convivencia, la tolerancia, la solidaridad, el respeto, la responsabilidad y que, en definitiva, sea un cauce que sirva para favorecer las relaciones humanas en la vía pública." (OCIO, 2010).

Del mismo modo hace hincapié en el aspecto formador, fijando como objetivo la formación del educando para que sepa comportarse de manera responsable en la vía pública tanto como peatón, como cuando utiliza la bicicleta, el ciclomotor o conduce automóviles. (OCIO, 2010).

Esta visión ha evolucionado bajo recomendaciones y regulaciones limitadas al aprendizaje de las normas de circulación. Hoy en día la educación vial se incluye en numerosas disciplinas como recurso para paliar las problemáticas sociales, medioambientales, y económicas, entre otras, derivadas de los accidentes de tráfico. A los efectos ecológico-ambientales de la movilidad es preciso sumar los sociales, sin olvidar los económicos producidos como consecuencia de los dos anteriores (MURGA, 2010). 
Igualmente se resaltan una serie de condiciones que debería reunir una educación de calidad en el marco de la educación vial. Algunos de estos requisitos suponen la ampliación de la enseñanza a todos los sujetos, la valía de los contenidos, la veracidad, utilidad y actualidad de lo que se enseña, la adaptación a los sujetos aprendices y la ampliación de la enseñanza a toda la vida y a todas las dimensiones de la vida (ZABALZA, 2010).

Desde esta perspectiva, procedemos a analizar las normativas relacionadas con el tránsito vial y las específicas del sistema escolar en España que aluden a la educación vial, evaluando cada una de ellas para reforzar la legislación vial en España y servir de referente a otros países.

\section{Regulación vial en España}

Nos centramos en la legislación para observar la evolución del concepto de educación vial. Se analiza la regulación vial en España desde el Código de Circulación de 1934 hasta la Regulación del Permiso y la Licencia de Conducción por Puntos de 2005.

La primera norma general dictada en España para regular el fenómeno del tráfico, se aprobó el 17 de septiembre del año 1900 (1899 es el año del fenómeno automovilístico en España), originando el Reglamento para el Servicio de Coches Automóviles por las Carreteras del Estado.

\subsection{4: Código de Circulación}

Hasta el Decreto de 25 de septiembre de 1934 y sus anexos, no se aprueba el Código de la Circulación que suponía el reconocimiento definitivo del fenómeno de la circulación. Aunque con anterioridad se habían publicado los Reglamentos de 1926 y 1928.

En el Artículo 7 se prescribe la obligatoriedad de impartir enseñanzas en los centros escolares, sobre normas e instrucciones y consejos de prudencia en relación con el tráfico.

El 275 fija las bases del aprendizaje de la conducción y la obtención de la licencia de aprendizaje (existe una gran similitud con nuestra normativa actual). Este código considera por primera vez la información, la formación y la educación vial de los ciudadanos. 


\subsection{9: Creación de la Dirección General de Tráfico}

Veinticinco años más tarde, se aprueba la Ley de Regulación de la Competencia en Materia de Tráfico (ESPAÑA, 1959) creándose la Dirección General de Tráfico (DGT en lo sucesivo) que desde entonces es el principal organismo regulador del fenómeno del tráfico en España.

Al igual que sucedió en Estados Unidos y en gran parte de Europa, el fenómeno del tráfico sufrió una profunda reestructuración, habida cuenta del aumento experimentado por el parque de automóviles.

Ni la gestión, ni la red de carreteras podían absorber semejante crecimiento. El lado más negativo de este desarrollo español lo constituyó el incremento en paralelo del número de accidentes (sólo en 1964 el número ascendería a 61.276). La Ley de 30 de julio de 1959 fue un verdadero hito, permitiendo aunar pasado y presente.

La sociedad se involucra cada vez más, haciendo suyo el fenómeno del tráfico, concienciándose sobre la necesidad de fomentar la educación vial, concebida como una educación integral que desarrolla el respeto y cuidado de la vida propia y de los demás.

\subsection{8: Constitución Española}

En la Constitución de 1978 aparecen también aspectos relacionados con la seguridad vial. En su Artículo 149.1.21, la Carta Magna atribuye al Estado las competencias en materia de "tráfico y circulación de vehículos a motor" con carácter de exclusividad. Esta atribución competencial es debida a la repercusión del tráfico en diversos ámbitos de la sociedad y a la necesidad de establecer una regulación uniforme a nivel autonómico.

\subsection{9: Ley de Bases sobre Tráfico, Circulación de Vehículos a motor y Seguridad Vial}

Conocida como la Ley de Bases sobre Tráfico, Circulación de Vehículos a motor y Seguridad Vial (ESPAÑA, 1989), en lo relativo a la educación vial, el objetivo primordial es que la sociedad conozca las consecuencias de los accidentes de tráfico y modifique sus comportamientos nocivos.

Tras la reforma del Código Penal en Seguridad Vial, aprobada el 7 de noviembre de 2007 y publicada en el Boletín Oficial de las Cortes Generales (BOCG) el 22 de junio 
de 2007, se modifica la Ley Orgánica 10/1995, de 23 de noviembre, del Código Penal en materia de Seguridad Vial en España 122/000222 (ESPAÑA, 2007), que además de elevar las faltas a delitos y poder acudir a la cárcel por incumplimiento de la normativa, manifiesta su papel formador, como herramienta más duradera y eficaz.

\section{2005: Regulación del Permiso y la Licencia de Conducción por Puntos}

Gracias a los resultados positivos en la Unión Europea del Permiso por Puntos, reduciendo de media un $10 \%$ el número de accidentes de tráfico en un año, ha servido de referente a países como España.

En España fue introducido el 1 de julio de 2006, por la Ley 17/2005, de 19 de julio (ESPAÑA, 2005a), en la que se regula el permiso y la licencia de conducción por puntos y se modifica el texto articulado de la Ley sobre tráfico, circulación de vehículos a motor y seguridad vial.

Se aprobó la Orden INT/2596/2005, de 28 de julio (ESPAÑA, 2005b), que determina el contenido de los cursos para recuperar puntos, o para recuperar el permiso o la licencia de conducción, en caso de agotarlos.

En el apartado dos se presentan los objetivos principales:

a) Sensibilizar a los participantes sobre las graves consecuencias humanas, económicas y sociales que se derivan de los accidentes de tráfico y sobre la especial implicación de los propios conductores en la producción de éstos.

b) Reeducar los comportamientos y actitudes de los participantes hacia la cultura de la seguridad vial y hacia unos valores de convivencia y respeto en el entorno del tráfico.

El pilar sobre el que se sustenta este dispositivo ya no es exclusivamente la sanción económica sino la formación.

\section{Educación vial y sistema escolar español}

Este apartado realiza un recorrido histórico sobre las leyes orgánicas de educación y el enfoque que otorgan a la formación vial.

La perspectiva de la educación vial en España desde el ámbito formal está marcada, al menos, por tres factores: 
- La actuación de las Administraciones.

- La actitud de los profesores y el desarrollo del currículum.

- El desarrollo socio-económico del país.

Las primeras referencias legislativas al respecto se sitúan en 1934, donde ya se recoge la obligatoriedad de la enseñanza de las normas relativas a la Seguridad vial en las Escuelas de Enseñanza Primaria. En la década de los 60 encontramos nuevos textos legales. En este sentido destacaríamos la orden ministerial de 1961, que establece las bases de creación, reglamentación y funcionamiento de los Parques Infantiles de Tráfico, como complemento educativo en la enseñanza de la educación vial.

Los cambios económicos y sociales producidos en la década de los 60 dieron lugar a una sociedad predominantemente urbana, y aceleradamente industrializada, a cuyas necesidades no podía responder el viejo sistema educativo.

\section{Ley General 14/1970, de 4 de agosto, de Educación y Financiamiento de la Reforma Educativa}

Esta ley, recogida en el BOE 187, de 6/8/1970, supuso un importante cambio en la incorporación de contenidos, propuestas metodológicas y desarrollo de la educación vial en la escuela. En 1973, como consecuencia de dicha ley, la Dirección General de Ordenación Educativa aprueba las Orientaciones Pedagógicas para el desarrollo de la educación vial en los Ciclos Inicial, Medio y Superior de la Educación General Básica (EGB), que supone un nuevo impulso a la temática en esta etapa. El artículo 127 de la ley garantiza el derecho del estudiante de cualquier nivel, a recibir la orientación educativa y profesional que precise. La educación vial no era un tema central si bien conoció desarrollos interesantes.

En 1980, con la publicación de los Programas Renovados, se pretendía modernizar y adaptar los contenidos de la Ley General de Educación de 1970. En ellos se proponen unas enseñanzas mínimas (Niveles Básicos de Referencia), alegando que los objetivos y contenidos de dicha ley eran excesivamente amplios y generales, en consecuencia se especificaban las Áreas de Conocimiento para cada ciclo (Inicial, Medio y Superior) donde se detallaban con mayor claridad, los objetivos, contenidos y actividades de cada curso. De igual modo, 
en estos programas se exponen los relativos a la educación vial, comenzando a tratarse desde una visión transversal de los contenidos, que completaría la Ley General de Educación de 1990.

\section{Ley Orgánica $1 / 1990$ de 3 de Octubre de Ordenación General del Sistema Educativo}

Tras la estela de la Ley Orgánica 8/1985, de 3 de julio, reguladora del Derecho a la Educación (ESPAÑA, 1985), la Ley Orgánica 1/1990 de 3 de Octubre de Ordenación General del Sistema Educativo (ESPAÑA, 1990), refleja la transversalidad de sus antecesoras pero de forma más explícita. Igualmente presenta los objetivos de educación vial en función de los comportamientos viales y del desarrollo evolutivo de los estudiantes.

Las áreas transversales son definidas por primera vez en los Diseños Curriculares Base (DCB) de 1989, como: "aspectos de especial relevancia para el desarrollo de la sociedad que deben integrar la actividad educativa en su conjunto". Consecuentemente la educación vial queda integrada junto con otras disciplinas en las diferentes disposiciones de la LOGSE. Así en el Real Decreto 1344/1991, de 6 de septiembre, por el que se establece el currículo de la educación primaria (ESPAÑA, 1991) se hace referencia a ella:

"El carácter integral del currículo significa también que a él se incorporan elementos educativos básicos que han de integrarse en las diferentes áreas y que la sociedad demanda, tales como la educación para la paz, para la salud, para la igualdad entre los sexos, educación ambiental, educación sexual, educación del consumidor y EDUCACIÓN VIAL". (ESPAÑA, 1991).

Esta legislación no se concretó íntegramente en la práctica, quedando reducida a clases esporádicas, pero es en ese contexto donde se produce una coyuntura histórica en España que permite mayor inserción de la educación vial, a nivel de legislación educativa y principalmente de orientaciones didácticas al profesorado.

En la Ley Orgánica 9/1995 de 20 de noviembre de la Participación, Evaluación y Gobierno de los centros docentes (LOPEG) se continúa el interés por la transversalidad en educación vial seguido desde la LOGSE. 
Con la llegada de la Ley Orgánica 10/2002, de 23 de diciembre, de Calidad de la Educación (ESPAÑA, 2002) apenas varía el trato que recibe esta temática con sus leyes precedentes.

En el capítulo I del Título Preliminar, el Artículo 1.b señalaba como uno de los principios de calidad del sistema educativo, la capacidad de transmitir valores trasladables a la educación vial.

La LOCE acentúa el tratamiento de la educación vial como eje transversal del currículo iniciado en la LOGSE, emparejando aspectos teóricos y prácticos, con una presencia continua en el sistema educativo, comprendiendo el ciclo vital de los ciudadanos. Esta ley se paralizó por el Consejo de Ministros, del 28 de mayo de 2004, siendo derogada por la entrada de su sucesora.

\section{Ley Orgánica de Educación 2/2006, de 3 de mayo, BOE 4/5/2006}

El planteamiento de esta ley se encamina hacia la transmisión de valores trasladables a la educación vial, aspecto admitido en la sociedad actual española, donde la educación vial recibe un tratamiento sustantivo, integrándose como contenido curricular dentro de la asignatura de Educación para la Ciudadanía.

Los accidentes de tráfico en su mayoría no se producen por un desconocimiento de la normativa sino por su incumplimiento, ante ello se piensa en la educación como contrapeso fundamental para dotar de sentido ético a la persona y a su interacción con el entorno (JIMÉNEZ, 2010a).

Desde el Preámbulo de esta Ley Orgánica de Educación (LOE), se promueve el aprendizaje de los valores democráticos. Los conocimientos no tienen sentido en sí mismos, sino en función de la adquisición de hábitos y actitudes, valiosos para el individuo y la sociedad:

"Su finalidad consiste en ofrecer a todos los estudiantes un espacio de reflexión, análisis y estudio acerca de las características fundamentales y el funcionamiento de un régimen democrático, de los principios y derechos establecidos en la Constitución española y en los tratados y las declaraciones universales de los derechos humanos [...]. La nueva materia permitirá profundizar 
en algunos aspectos relativos a nuestra vida en común, contribuyendo a formar a los nuevos ciudadanos." (ESPAÑA, 2006).

Con anterioridad a la LOE, el término vial en España era entendido exclusivamente como el aprendizaje de normas y señales de tráfico, aislando aspectos referidos al aprendizaje de conocimientos, destrezas y valores. Esta ley se inspira en el compromiso de la Unión Europea, por implantar objetivos educativos en la escuela y por la evolución acelerada de la ciencia y la tecnología y el impacto que dicha evolución tiene en el desarrollo social.

Según la LOE, los cursos en los que se imparte educación vial son:

\section{Primaria:}

En el Capítulo II, Artículo 16: "La educación primaria es una etapa educativa que comprende seis cursos académicos, que se cursarán ordinariamente entre los seis y los doce años de edad".

En su Artículo 17: Objetivos de la educación primaria. El apartado n, destaca: "Fomentar la educación vial y actitudes de respeto que incidan en la prevención de los accidentes de tráfico".

El Artículo 18: Organización. En el apartado 1: La educación primaria comprende tres ciclos de dos años académicos cada uno y se organiza en áreas con carácter global e integrador.

En el punto 2 se mencionan esas áreas (por ejemplo: conocimiento del medio natural, social y cultural).

El punto 3 señala que en uno de los cursos del tercer ciclo de la etapa $\left(5^{\circ}\right.$ ó $\left.6^{\circ}\right)$, se añade el área de educación para la ciudadanía y los derechos humanos.

Los contenidos de la asignatura se imparten en Primaria en tres bloques formativos. El titulado "Vivir en sociedad", plantea cuestiones como: Protección civil, seguridad, defensa -como compromiso cívico y solidario al servicio de la paz- y educación vial, profundizando en su papel en el contexto internacional. 


\section{Secundaria:}

La etapa de Educación Secundaria Obligatoria (ESO) recogida en el Capítulo III de la LOE, comprende cuatro cursos (ordinariamente, entre los doce y los dieciséis años de edad).

Clasificados en dos etapas:

- Secundaria, $1^{\circ}, 2^{\circ}$ y $3^{\circ}$ de ESO:

En la ESO tiene distinta denominación, pero parecidos contenidos: Educación para la Ciudadanía y los Derechos Humanos. Impartida en uno de los tres primeros cursos de secundaria (Punto 3, Artículo 24).

Se estructura en cinco bloques y apuesta por: la dimensión humana, respeto y cuidado del medio ambiente, circulación vial y consumo racional. Se realizan debates, exposiciones, evaluación crítica y búsqueda de información. Los criterios de evaluación se centran en estos contenidos.

El bloque IV: "Las Sociedades democráticas del siglo XXI", menciona la circulación vial y la responsabilidad ciudadana. Accidentes de circulación: causas y consecuencias.

- Secundaria, $4^{\circ}$ ESO

Denominada Educación ético cívica (Artículo 25 de la LOE), pretende formar sobre los interrogantes del ser humano, los sentimientos y emociones en las relaciones interpersonales y las teorías éticas, entre otros.

Consta de seis bloques, entre ellos: Bloque II: Identidad y alteridad. Educación afectivo-emocional; Bloque III: Teorías éticas. Los derechos humanos; Bloque V: Problemas sociales del mundo actual.

\section{Bachillerato:}

Los principios generales (Artículo 32) del Capítulo IV de la LOE, reflejan que: Podrán acceder a los estudios de bachillerato los alumnos que estén en posesión del título de Graduado en Educación Secundaria Obligatoria. Comprende dos cursos y se organiza flexiblemente, acorde con las perspectivas e intereses de los estudiantes. Podrán permanecer en régimen ordinario durante cuatro años. 
Los objetivos de dicha etapa (Artículo 33), afianzados en el punto n, señalan las actitudes de respeto y prevención en el ámbito de la seguridad vial (ubicada en la materia de Filosofía y Ciudadanía).

Incluir educación vial como materia obligatoria en colegios e institutos, destaca su función socializadora como lugar privilegiado para inculcar valores a edades vulnerables (menores y jóvenes).

Estas promulgaciones plantean nuevos retos a los gobiernos, instituciones y planes de estudios dirigidos al futuro profesorado. Ubicándonos en los principales centros en España que forman a los maestros en educación vial, señalamos: La Escuela Ausiàs March de la Universidad de Valencia, que diseñó asignaturas con este instituto, la Universidad de Salamanca (Centro Superior de Salamanca de educación vial) y la Universidad de Málaga. Estos centros enseñan a los futuros formadores educación vial para que la transmitan a sus estudiantes. Desde otras universidades, como la Universidad Nacional de Educación a Distancia (UNED) se imparten cursos, principalmente dirigidos a orientadores y profesores, para formarles sobre educación vial, quienes trasladan la necesidad de esta temática a su ámbito laboral. En 2009 desde la UNED se propone la educación vial como una asignatura optativa para los títulos de Grado de Educación Infantil, Educación Social y Pedagogía.

Al Instituto Universitario de Tráfico y seguridad vial (INTRAS, Universidad de Valencia), centro en España colaborador en aspectos como la puesta en marcha del carné por puntos, se le solicita en 2002 y por petición de Magisterio, la asignatura de educación vial como materia optativa. Del mismo modo existen dos asignaturas sobre tráfico en la carrera de Psicología de dicha universidad. El peso de esta formación lleva años recayendo también en otros organismos como ministerios, asociaciones, entidades, clubes, fundaciones, empresas, y universidades. Como hemos indicado, la educación vial es introducida en los programas de grado que estipula la Unión Europea, donde comienza a tener una fuerza sustancial.

Desde una perspectiva internacional, las investigaciones y estadísticas relativas a los accidentes provocados por el fenómeno circulatorio, nos muestran que éstos constituyen uno de los problemas más importantes de salud pública (OMS, 2004). En este sentido, la circulación vial se orienta como un tema social que nos afecta y compete a todos, y que demanda la toma de conciencia de los 
riesgos y problemas que ocasiona, para que, desde la prevención, puedan encontrarse soluciones y comience a existir una mayor sociabilidad vial.

\section{Conclusiones}

A pesar de que la creación del vehículo ha supuesto un progreso indudable para la evolución de las sociedades, éste presenta al mismo tiempo unas desventajas elevadas tales como, la destrucción ecológica y el número de fallecidos en las carreteras. Estos acontecimientos plantean a la sociedad nuevas normativas que permitan reducir estos riesgos.

La legislación ha ido adecuándose a los cambios políticos, culturales, educativos y sociales acaecidos a lo largo de los años. Debido al aumento de los accidentes de tráfico, actualmente ha sido imprescindible la inclusión de la educación vial en las normativas viales propiamente dichas, y en las educativas, para suscitar actitudes y comportamientos seguros y cívicos, facilitando los medios precisos para adecuar los aprendizajes a las características y necesidades de los usuarios de transportes, peatones, conductores y viajeros.

La evolución legislativa analizada y la creciente incorporación de la educación vial a nivel formativo en los centros educativos, desde la infancia hasta la universidad, plantea nuevos desafíos a educadores e instituciones. Pero no sólo se enfatiza la responsabilidad de docentes e instituciones, sino que además se acentúa el compromiso civil donde cada ciudadano se forma y se conciencia ante sus prácticas cotidianas viales y sirve de modelo a los demás.

La prospectiva se encamina hacia una educación vial que enfatiza la necesidad de inculcarla desde la infancia, ya que las primeras relaciones del niño con el entorno determinarán su actuación futura. Al mismo tiempo se resalta su continuidad en el tiempo, fomentando el aprendizaje a lo largo de la vida desde una perspectiva de aprendizaje de valores, que forme ciudadanos cívicos y garantice la durabilidad de los comportamientos adecuados.

Se resalta también la necesidad de la homogeneización de la información entre los diferentes países, que permita una actualización de los datos y una evaluación de los costes y beneficios de ciertas intervenciones, decidiendo sobre cuáles constituyen una mejor inversión y cuáles no. 
A pesar de la evolución que muestra este repaso normativo, la realidad es que la educación vial no ha llegado a ser aún un área de conocimiento propiamente dicho dentro del sistema educativo español, pero está experimentando un cambio sustancial. Este cambio se debe a que España se guía por países con una larga trayectoria en la implantación de educación vial en sus programas educativos y de seguridad vial y que han visto reducir sus cifras de fallecidos en las carreteras. Las normas de tráfico venían entendiéndose como dependientes de la autoridad, por lo que se cumplían para no ser sancionados. Posteriormente, dichas normas comienzan a percibirse como elementos indispensables para satisfacer necesidades propias y para convivir en sociedad.

En este marco la educación vial se percibe en la actualidad como una educación sin límites de edad, flexible, que contribuye a la socialización y al desarrollo de las personas, integrada en el medio, que atiende a contextos políticos, socioeducativos, medioambientales, sanitarios, entre otros, en los que se promueve un aprendizaje vial eficaz.

\section{Referências}

ESPAÑA. Ministerio de Educación. Establece el currículo de la Educación Primaria. Boletín Oficial del Estado, n. 220, de 6 set. 1991, Sec. 1, p. 30266 30228 .

. Ministerio de la Presidencia. Ley de bases $n{ }^{\circ} 18$, de 25 de julio de 1989. Sobre tráfico, circulación de vehículos a motor y seguridad vial. Boletín Oficial del Estado, n. 178, 27 jul. 1989.

. Ministerio del Interior. Ley $n^{\circ} 47$, de 30 de julio de 1959. Regulación de la competencia en materia de tráfico. Boletín Oficial del Estado, [s.n.], jul. 1959.

. Ministerio de Educación. Ley orgánica $n^{\circ} 10$, de 23 de diciembre de 2002. Calidad de la educación. Boletín Oficial del Estado, n. 307 de 24 dic. 2002, Sec. 1, p. 45188 - 45220. Disponível em: <http://www.boe.es/ boe/dias/2002/12/24/pdfs/A45188-45220.pdf> Acesso en: 15 nov. 2011. 
ESPAÑA. Ministerio de Educación. Ley orgánica $n^{\circ}$ 8, de 3 de julio de 1985 reguladora del derecho a la educación. Boletín Oficial del Estado, n. 251 de 19 enero 1985, p. 33013. Disponível em: $<$ http://www.mec.es/mecd/atencion/educacion/hojas/E_SistemaEduc/e-1-3.htm> Acesso em: 15 nov. 2011.

. Ministerio de Educación. Ley orgánica $\mathrm{n}^{\circ} 1$ de 3 de octubre de 1990. Ordenación general del sistema educativo. Boletín Oficial del Estado, n. 238 de 4 oct. 1990, p. 28927.

. Ministerio de la Presidencia. Ley orgánica ${ }^{\circ} 17$, de 19 de julio de 2005. Permiso y la licencia de conducción por puntos. Modificación del texto articulado de la ley sobre tráfico, circulación de vehículos a motor y seguridad vial. Boletín Oficial del Estado, $\mathrm{n}^{\mathrm{o}} 172$ de 20 jul. 2005a, p. 25781 - 25793.

. Ministerio de la Presidencia. Orden interna ${ }^{\circ}{ }^{\circ} 2596$, de 28 de julio de 2005. Por la que se regulan los cursos de sensibilización y reeducación vial para los titulares de un permiso o licencia de conducción. Boletín Oficial del Estado, n. 190 de 10 agosto 2005b, p. 28083 - 28087.

. Ministerio de Justicia. Ley $\mathrm{n}^{\circ} 122 / 000222$ de la reforma del código penal en seguridad vial, por la que se modifica la ley orgánica 10/1995, de 23 de noviembre, del código penal en materia de seguridad vial. Boletín oficial de las cortes generales, 283-1 de 22 de jun. de 2007. Disponível em: $<\mathrm{http} / / /$ www.congreso.es/public_oficiales/L8/CONG/BOCG/B/B_283-01.PDF> Acesso em: 23 sept. 2011.

. Ministerio de Educación. Ley orgánica n. 2, de 3 de mayo de 2006 educación. Boletín Oficial del Estado, n. 106, de 04 mayo 2006, Sec. 1, p. $17158-17207$.

JIMÉNEZ FERNÁNDEZ, C. Educación vial, respuesta a una necesidad social. Barcelona: Editorial Davinci, 2010a.

JIMÉNEZ FERNÁNDEZ, C. Educación vial, una necesidad social. In: JIMÉNEZ FERNÁNDEZ, C. (Coord.): Educación vial, respuesta a una necesidad social. Barcelona: Editorial Davinci, p. 15-40, 2010 b. 
MANSO, V.; CASTAÑO, M. Educación y Seguridad Vial: la aportación de los agentes sociales en la movilidad segura. Madrid: Editorial Atrasa S.A, 2008.

MURGA, M. A. La movilidad sostenible: un objetivo de la educación vial en el contexto europeo. In: JIMÉNEZ FERNÁNDEZ, C. (Coord.). Educación vial, seguridad vial. Barcelona: Editorial Davinci, p. 65-91, 2010.

OCIO, E. La educación vial en España: marco histórico legal. In: JIMÉNEZ FERNÁNDEZ, C. (Coord.): Educación vial, seguridad vial. Barcelona: Editorial Davinci, p. 143-161, 2010.

WERLE, F.; SOARES, J. Plano Municipal de Educação e a afirmação de principios para a educação local. Ensaio: aval.pol.públ. Educ. , Rio de Janeiro, v. 16, n. 61, p. 515-542, 2008. Disponível em: <http://www.scielo.br/pdf/ensaio/ v16n61/v16n61a03.pdf> Acesso em: 13 sep. 2011.

ZABALZA, M. Á. La educación vial en el marco de una formación por competencias. En JIMÉNEZ FERNÁNDEZ, C. (Coord.). Educación vial, seguridad vial. Barcelona: Editorial Davinci, p. 162-184. 2010.

Recebido em: 02/02/2012

Aceito para publicação em: 27/03/2012 


\section{Evolução legislativa da educação viária na Espanha: novos desafios para educadores e instituições Resumo}

A educação viária sofreu variações ao longo dos anos. De um conceito restringido a aspectos de circulação e normas, derivou-se a uma conscientização cidadã que engloba aspectos legais, do meio ambiente, médicos, econômicos, pessoais e convencionais, entre outros.

Desde o Dia Mundial da Saúde celebrado em 2004 e dos seguintes debates na Assembleia Geral das Nações Unidas, os Governos se interessam cada vez mais pela Segurança Viária, insistindo na necessidade da formação viária, como meio para reduzir os acidentes de trânsito.

A presente colaboração trata sobre a regulação viária na Espanha, analisando a incorporação da educação viária no currículo e sua evolução desde o Código de Circulação de 1934 até a Lei Orgânica de Educação, e oferece novos desafios e obrigações aos já enfrentados pelos governos, educadores e instituições, com o propósito de diminuir os elevados índices de acidentes e vítimas nas estradas.

Palavras-chave: Educação viária. Legislação. Cidadania.

\section{Legislative development of road education in Spain: new challenges for teachers and institutions Abstract}

Road education has changed over the years. From a conception restricted to aspects related to traffic and regulations, it has resulted in a public awareness that includes legal, environmental, medical, economic, personal and coexistence aspects, among others. Since World Health Day held in 2004 and the subsequent discussions in the General Assembly of United Nations, governments are increasingly interested in Road Safety, stressing the need to road training as a means to reduce traffic accidents. This contribution analyzes the road regulation in Spain, putting the emphasis on the incorporation of road education in the curriculum and its development from the Highway Code of 1934 to the fundamental law of Education, and it offers new challenges and obligations to which governments, teachers and institutions face up to try to curb the high accident rate on the road.

Keywords: Education. Road. Legislation. Citizenship. 